\title{
International Medical Terminology
}

National Cancer Institute

\section{Source}

National Cancer Institute. International Medical Terminology. NCI Thesaurus. Code C43557.

The International Medical Terminology is a clinically validated standard terminology for the reporting of regulatory activities, originally based upon the Medical Dictionary for Drug Regulatory Affairs (MedDRA), developed under the auspices of the International Conference on Harmonization of Technical Requirements for Reg istration of Pharmaceuticals for Human Use (ICH). 